\title{
Complicaciones de la nefrolitotomía percutánea según la clasificación de Clavien-Dindo modificada. Experiencia institucional
}

López-Maguey RP, Gómez-Sánchez J, Martínez-Arroyo C, Herrara-Muñoz J, Sánchez-Aquino $U$, y colaboradores.

\section{Resumen}

ANTECEDENTES: la incidencia y prevalencia de la litiasis urinaria se han incrementado a nivel mundial. Algunos estudios reportan una prevalencia de $13 \%$ en el área de hospitalización por enfermedad renal. La nefrolitotomía percutánea es un procedimiento de referencia para extraer cálculos renales mayores de $2 \mathrm{~cm}$. La clasificación de Clavien-Dindo se ha propuesto para graduar las complicaciones provocadas por la nefrolitotomía percutánea.

OBJETIVO: determinar la prevalencia y los factores de riesgo asociados con complicaciones de la nefrolitotomía percutánea.

MATERIALES Y MÉTODOS: estudio retrospectivo y descriptivo efectuado mediante la revisión de los expedientes de pacientes a quienes se realizó nefrolitotomía percutánea entre los años 2013 y 2016. Variables analizadas: comorbilidades, índice de masa corporal (IMC), volumen litiásico y complicaciones perioperatorias. Para el análisis estadístico se utilizó el programa SPSS ${ }^{\circledR}$ 2.0.

RESULTADOS: se practicaron 47 nefrolitotomías percutáneas; las comorbilidades identificadas fueron hipertensión en 12 (25\%) pacientes y diabetes en 16 (34\%). El IMC promedio fue de $28.7 \mathrm{~kg} / \mathrm{m}^{2}$ y el volumen litiásico de $446 \mathrm{~mm}^{3}$. Según la clasificación de Clavien-Dindo las complicaciones más frecuentes $(7[14 \%])$ correspondieron a grado II (71\%) y grado IIla (28\%). Las comorbilidades, IMC y volumen litiásico no fueron estadísticamente significativas para sufrir complicaciones ( $p=0.197, p=0.551, p=0.185$, respectivamente).

CONCLUSIONES: las comorbilidades, IMC y volumen litiásico no se relacionan con complicaciones posquirúrgicas de la nefrolitotomía percutánea.

PALABRAS CLAVE: nefrolitotomía percutánea, clasificación de Clavien-Dindo, litiasis urinaria
División de Urología, Hospital General Dr. Manuel Gea González, Ciudad de México.

Recibido: diciembre 2016

Aceptado: julio 2017

Correspondencia

Dr. Roberto Patricio López Maguey

rpatloma@gmail.com

Este artículo debe citarse como

López-Maguey RP, Gómez-Sánchez J, MartínezArroyo C, y col. Complicaciones de la nefrolitotomía percutánea según la clasificación de Clavien-Dindo modificada. Experiencia interinstitucional. Rev Mex Urol. 2017 jul-agos;77(4):272-278.

DOI: https://doi.org/10.24245/revmexurol.v77i4.1102 


\section{Percutaneous nephrolithotomy complications according to the modified Clavien-Dindo classification: An institutional experience}

López-Maguey RP, Gómez-Sánchez J, Martínez-Arroyo C, Herrara-Muñoz J, Sánchez-Aquino U, et al.

\begin{abstract}
BACKGROUND: The incidence and prevalence of urinary lithiasis has increased worldwide. Some studies report a prevalence of $13 \%$ of all hospitalizations for kidney disease. Percutaneous nephrolithotomy is a standard procedure for the extraction of kidney stones larger than $2 \mathrm{~cm}$. The Clavien-Dindo classification ranks the complications arising from that procedure.
\end{abstract}

OBJECTIVE: To determine the prevalence of and risk factors for complications in percutaneous nephrolithotomy.

MATERIALS AND METHODS: Retrospective and descriptive study carried out by the medical records of patients that underwent percutaneous nephrolithotomy within the time frame of 2013 to 2016 were reviewed. Comorbidities, body mass index, stone volume, and perioperative complications were evaluated and the SPSS ${ }$ program was used for the statistical analysis.

RESULTS: Forty-seven percutaneous nephrolithotomies were performed during the study period. The comorbidities identified were high blood pressure in 12 (25\%) patients and diabetes in $16(34 \%)$. The mean body mass index was $28.7 \mathrm{~kg} / \mathrm{m}^{2}$ and the mean stone volume was $446 \mathrm{~mm}^{3}$. The most frequent complications (7 [14\%]), according to the Clavien-Dindo classification, were grade II (71\%) and grade IIla $(28 \%)$. Comorbidities, body mass index, and stone volume were not statistically significant for the development of complications $(\mathrm{p}=$ $0.197, p=0.551, p=0.185$, respectively).

CONCLUSIONS: Comorbidities, such as obesity, have been shown in the international medical literature to be risk factors for the development of major complications in percutaneous nephrolithotomy. However, in our population, comorbidities, body mass index, and stone volume were not related to the development of complications.

KEYWORDS: Percutaneous nephrolithotomy; Clavien-Dindo; Urinary lithiasis
Urology Division, Hospital General Dr. Manuel Gea González, Mexico city, Mexico.

Correspondence

Dr. Roberto Patricio López Maguey rpatloma@Gmail.Com 


\section{ANTECEDENTES}

La incidencia y prevalencia de litiasis urinaria se han incrementado en todo el mundo. En México existen pocos estudios epidemiológicos de litiasis urinaria. Otero y su grupo reportan una prevalencia de $13 \%$ en el área de hospitalización por enfermedad renal en el Instituto Mexicano del Seguro Social. ${ }^{1}$ La dieta, cambios del $\mathrm{pH}$ urinario, hipercalciuria, hiperuricosuria, hiperoxaluria y características genéticas son factores de riesgo que aumentan la incidencia de la enfermedad. ${ }^{2}$

La nefrolitotomía percutánea se ha convertido en el procedimiento de referencia para extraer cálculos mayores de $2 \mathrm{~cm}$ de la pelvis renal o de $1.5 \mathrm{~cm}$ del área calicial (incluso ha desplazado a la cirugía abierta), por lo que las Guías de Práctica Clínica la recomiendan ampliamente. Desde su introducción, en 1976 por Fernström y Johansson, ha evolucionado considerablemente para mejorar la técnica quirúrgica, y de esta manera minimizar el dolor y la morbilidad perioperatoria. $^{3,4}$

La nefrolitotomía percutánea ha sufrido variaciones en la última década, por ejemplo, en Estados Unidos se ha incrementado su práctica en hospitales comunitarios, de 1999 a 2009, con mayor incidencia en mujeres. Aunque existe un aumento en el número de procedimientos relacionados con la litiasis urinaria, la proporción de nefrolitotomías percutáneas ha disminuido de $6 \%$ en 1995 a menos de $3.5 \%$ en $2010 .{ }^{4}$

Existen diversos factores implicados en la práctica de la nefrolitotomía percutánea; en países donde no se reutiliza el instrumental, este tipo de técnicas es mucho más alto. Aunque la nefrolitotomía percutánea no es una técnica tan común como la litotripsia extracorpórea por ondas de choque $(\mathrm{LEOCH})$, incluso como la ureteroscopia, sigue siendo el tratamiento de referencia para extraer litos complejos, además de considerarse un procedimiento mayor, con potencial de mortalidad significativa. ${ }^{5}$

La nefrolitotomía percutánea es un procedimiento no exento de complicaciones, en la era moderna se han estudiado la prevalencia y severidad de las complicaciones, por ejemplo: hemorragia, infecciones de la vía urinaria inferior, fiebre y sepsis. Los factores asociados con mayor riesgo de complicaciones incluyen: edad, sexo, tiempo quirúrgico y comorbilidades. En Estados Unidos se han incrementado las complicaciones relacionadas con la sepsis. Así mismo, se ha estimado que el aumento de readmisión posterior al procedimiento es de $15 \%$ en Estados Unidos, $12 \%$ en Canadá y $9 \%$ en el Reino Unido; sin embargo, la mortalidad sigue siendo una complicación excepcional $(0.2 \%){ }^{5}$

El objetivo de este estudio fue determinar la prevalencia y los factores de riesgo de complicaciones de la nefrolitotomía percutánea.

\section{MATERIALES Y MÉTODOS}

Estudio retrospectivo y descriptivo, efectuado mediante el análisis de los expedientes de pacientes con nefrolitotomía percutánea, atendidos en el Hospital General Dr. Manuel Gea González, entre mayo de 2013 y junio de 2016. De los expedientes se consideraron datos preoperatorios como: identificación del paciente, índice de masa corporal (IMC) y volumen litiásico. Se recolectaron datos de complicaciones intraoperatorias y persistencia de fragmentos objetivados durante la cirugía (Stone free rate [SFR]). Finalmente, se realizaron estudios de control posoperatorios, después de un mes de efectuar el procedimiento quirúrgico. 
Para el análisis de los datos se utilizó la prueba estadística de $\chi^{2}$, con la finalidad de determinar la incidencia de complicaciones, según la clasificación de Clavien-Dindo modificada. Los datos se analizaron en la base de datos SPSS $^{\circledR} 2.0$

El diagnóstico prequirúrgico, para justificar la nefrolitotomía percutánea, se estableció mediante TAC con contraste y reconstrucciones tridimensionales, para de esta manera realizar una planificación correcta de la cirugía, valorar la ventana de punción, dimensiones reales de la litiasis a tratar y los mejores cálices de acceso para la extracción de todo el cálculo en un solo procedimiento.

La masa litiásica se calculó a partir de la mayor área de sección transversal del cálculo identificado en el corte anteroposterior, medido en $\mathrm{mm}^{3}$ a partir de la tomografía preoperatoria. En cuanto a la técnica quirúrgica se realizó nefrolitotomía percutánea en posición supina, con previo cateterismo ureteral para contrastar la vía de acceso. Como método de punción se utilizó una aguja de $18 \mathrm{G}$ guiada por fluoroscopia. La dilatación se realizó con balón $X$-force ${ }^{\circledR}$. Se utilizó un nefroscopio Olympus y como energías de fragmentación, Lithoclast y láser holmio.

\section{RESULTADOS}

Se efectuaron 47 nefrolitotomías percutáneas. La edad promedio de los pacientes fue de 49 años; se identificaron $31 \%$ de cálculos en el lado derecho, $48 \%$ en el lado izquierdo, 12\% bilaterales y $9 \%$ en otro sitio. La distribución por sexo fue de 27 hombres y 20 mujeres. Entre los factores de riesgo de complicaciones se encontró: hipertensión en $12(25 \%)$ y diabetes mellitus en $16(34 \%)$ pacientes. El promedio de IMC fue de $28.7 \mathrm{~kg} / \mathrm{m}^{2}$ (Figura 1).

La dimensión media de las litiasis fue de 2.5 $\pm 0.4 \mathrm{~cm}^{2}$. La mayor parte de los cálculos se

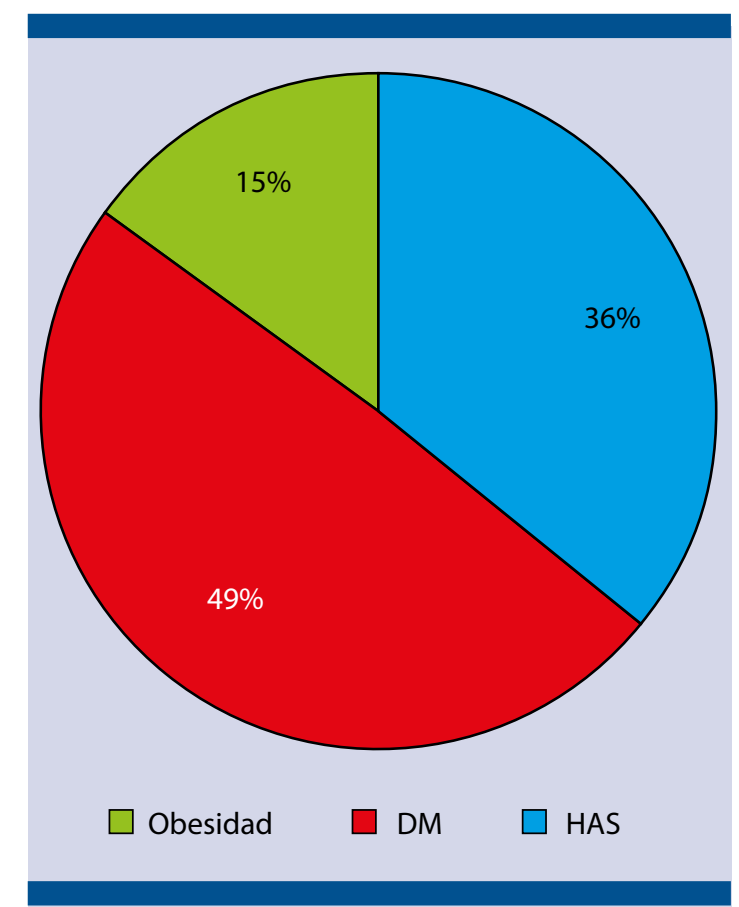

Figura 1. Distribución de comorbilidades.

DM: Diabetes mellitus; HAS: hipertensión arterial sistémica.

localizó en el cáliz inferior (31\%), seguidos de coraliformes incompletos (29\%), múltiples (más de 3 litos [21\%]) y la pelvis renal (19\%). De acuerdo con el objetivo principal de nuestro estudio, se registraron 7 (14\%) pacientes con complicaciones (Cuadro 1): grado I: $0 \%$, grado II: $71 \%$, grado IIla: $28 \%$ y grado IIlb: $0 \%$, según la clasificación de Clavien-Dindo modificada. No se observaron complicaciones relacionadas con los grados IVa, IVb o V (Figura 2).

La complicaciones más frecuentes fueron pielonefritis (los pacientes requirieron prescripción de carbapenémicos) y dolor, mientras que la complicación mayor fue fístula arteriovenosa, que requirió embolización selectiva, clasificada como Illb según el criterio Clavien-Dindo. No se observaron diferencias estadísticamente significativas para sufrir complicaciones al comparar las 
Cuadro 1. Graduación posoperatoria de complicaciones en pacientes con nefrolitotomía percutánea, según la clasificación de Clavien-Dindo modificada ${ }^{8}$

\section{Grado de complicación Descripción}

o Sin complicaciones.

I Desviación del curso posoperatorio normal, sin requerimiento de intervención.

II Complicación menor que requiere intervención farmacológica, incluida la trasfusión sanguínea o nutrición parenteral total.

Illa Complicaciones que requieren intervención quirúrgica, endoscópica o radiológica, pero de alivio espontáneo, sin necesidad de anestesia general

IIlb Complicaciones que requieren intervención quirúrgica, endoscópica o radiológica, pero de alivio espontáneo, con requerimiento de anestesia general.

IVa

Complicaciones que ponen en riesgo la vida y que requieren tratamiento en la unidad de terapia intensiva, con un solo tipo de disfunción orgánica, incluida la diálisis.

$\mathrm{IVb}$

Complicaciones que ponen en riesgo la vida y que requieren tratamiento en la unidad de terapia intensiva, con disfunción orgánica múltiple.

V Muerte provocada por la complicación.

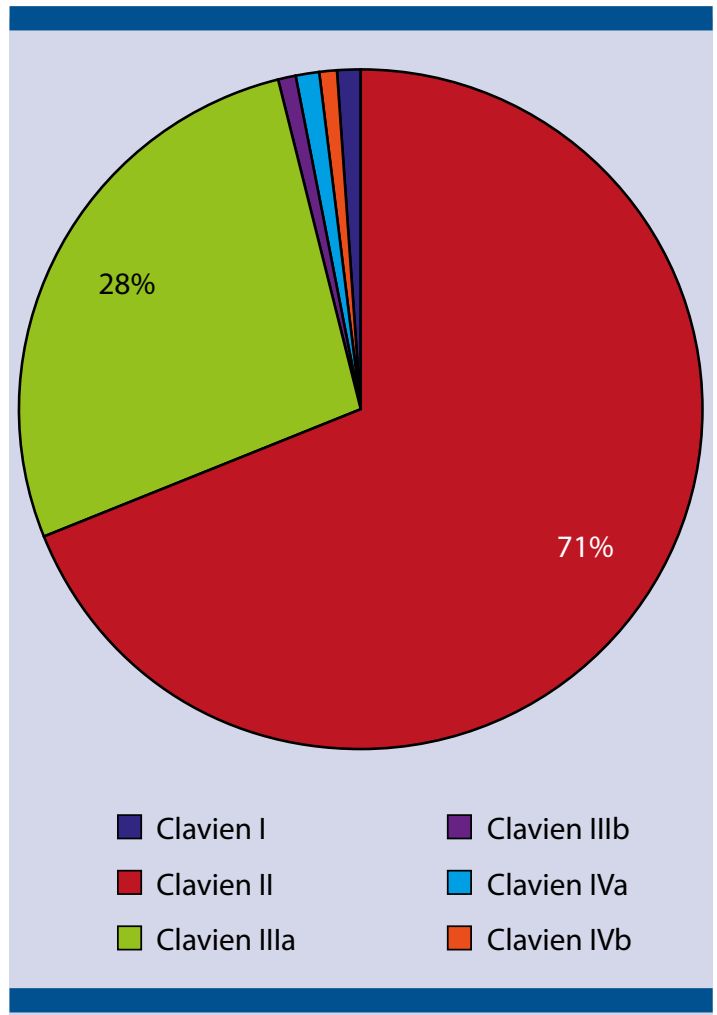

Figura 2. Distribución de complicaciones según la clasificación de Clavien-Dindo. comorbilidades, IMC y carga litiásica $(\mathrm{p}=0.197$, $p=0.551, p=0.185$, respectivamente; Cuadro 2).

\section{DISCUSIÓN}

La nefrolitotomía percutánea es un procedimiento que ha demostrado efectividad en el

Cuadro 2. Factores de riesgo de complicaciones por la nefrolitotomía percutánea

\begin{tabular}{|l|c|c|}
\hline Carga litiásica & $\begin{array}{c}\text { Complicaciones } \\
(\mathbf{n = 4 7})\end{array}$ & $\mathbf{P}$ \\
\hline $0-399 \mathrm{~mm}^{3}$ & $3(10.7 \%)$ & \\
\hline $400-799 \mathrm{~mm}^{3}$ & $2(16.7 \%)$ & 0.185 \\
\hline $800-1599 \mathrm{~mm}^{3}$ & $2(50 \%)$ & \\
$>16000 \mathrm{~mm}^{3}$ & - & \\
\hline Índice de masa corporal & & \\
$<20 \mathrm{~kg} / \mathrm{m}^{2}$ & $1(25 \%)$ & \\
\hline $21-30 \mathrm{~kg} / \mathrm{m}^{2}$ & $4(19 \%)$ & 0.551 \\
\hline$>31 \mathrm{~kg} / \mathrm{m}^{2}$ & $2(9.1 \%)$ & \\
\hline Comorbilidades & & \\
\hline Diabetes & $5(21.7 \%)$ & 0.197 \\
\hline Hipertensión arterial sistémica & $2(8.3 \%)$ & \\
\hline
\end{tabular}


tratamiento de casos complejos de litiasis renal. Aunque es una técnica mínimamente invasiva, no está exenta de complicaciones, por lo que es importante conocerla y clasificarla, además de determinar los factores predisponentes. ${ }^{1,7} \mathrm{En}$ 1992, el Dr. Clavien desarrolló una clasificación de complicaciones quirúrgicas, en la que describió como ventajas potenciales disminuir la morbilidad asociada con la técnica quirúrgica y los factores de riesgo y reconocer oportunamente las complicaciones. ${ }^{7}$ En 2004, el Dr. Dindo realizó 4 modificaciones importantes de la técnica para aumentar su confiabilidad. ${ }^{4,7}$ La primera modificación fue para disminuir las complicaciones que ponían en riesgo la vida del paciente, quienes requieren cuidados intermedios o intensivos, y se diferencian de las complicaciones atendidas en la sala de hospitalización. En segundo lugar, se consideran todas las complicaciones que afectan el sistema nervioso central en la misma categoría (complicaciones grado IV). En tercer lugar, el tiempo de estancia hospitalaria no se asocia con complicaciones. Y en cuarto lugar, la eliminación de la incapacidad como un grado en sí mismo.

En 2012, la CROES (Clinical Research Office of the Endourological Society) realizó una adaptación de la clasificación para la práctica de la nefrolitotomía percutánea. Un estudio efectuado en 5803 pacientes sometidos a este procedimiento, que evaluó la clasificación de las complicaciones (variables: comorbilidades índice de masa corporal, tiempo quirúrgico, volumen litiásico, ASA y anormalidades anatómicas), reportó principalmente hemorragia e infección por cultivos positivos, además de mayor riesgo conforme más prolongado fue el tiempo quirúrgico (115 minutos o más). ${ }^{8}$ En el estudio aquí reportado la principal complicación fue la pielonefritis con cultivos multirresistentes, cuyos pacientes ameritaron más días de hospitalización y reingreso. En cuanto a la obesidad se observó menor tasa libre de litiasis, variable relacionada estrecha- mente con las reintervenciones, sobre todo en pacientes con índice de masa corporal mayor de $40 \mathrm{~kg} / \mathrm{m}^{2} ; 9$ sin embargo, en este estudio no se encontró diferencia estadísticamente significativa relacionada con el IMC, quizá debido al tamaño pequeño de la muestra (solo se observó en $9 \%$ de los pacientes con obesidad).

El estudio de Ceballos-López y su grupo señala resultados similares a los obtenidos en otras investigaciones; por ejemplo, su evaluación en 141 pacientes reportó como principal complicación el grado I de Clavien y solo $21 \%$ de los pacientes requirió intervención quirúrgica. ${ }^{10}$ En el estudio aquí reportado, un paciente requirió radiología intervencionista debido a una fístula, lo que es un caso excepcional. La incidencia de punción intestinal también es muy baja.

Hoy en día la clasificación de Clavien-Dindo modificada es la técnica más útil para evaluar las complicaciones posquirúrgicas.

\section{CONCLUSIONES}

La clasificación de Clavien-Dindo es de gran utilidad para estadificar adecuadamente las complicaciones posoperatorias en pacientes sometidos nefrolitotomía percutánea. En nuestra población, las comorbilidades, IMC y volumen litiásico no se relacionan con complicaciones posquirúrgicas; sin embargo, se requieren estudios adicionales, con mayor número de pacientes, para ampliar esta información.

\section{Financiamiento}

No se recibió ningún tipo de financiamiento para realizar este trabajo.

\section{Conflicto de intereses}

Los autores declaran no tener conflictos de interés. 


\section{REFERENCIAS}

1. Otero F, Lugo A, Durán A. Las enfermedades renales en el Instituto Mexicano del Seguro Social (1982-1989). Rev Asoc Med Int Mex. 1995;11:21-29.

2. Khurshi R, Sero A. Et al. Percutaneous nephrolithotomy: update trends and future directions. Eur Urol, 2016;6647:1-15.

3. Türk C, Knoll T, Petrik A, et al. Guidelines on urolithiasis. European Association of Urology Web site.http://uroweb.org/ wp-content/uploads/22-Urolithiasis_LR.pdf. Updated 2014.

4. Preminger GM, Assimos DG, Lingeman JE, Nakada SY, Pearle MS, Wolf JS. Chapter 1: AUA guideline on management of staghorn calculi: diagnosis and treatment recommendations. J Urol, 2005;173:1991-2000.

5. Fernström I, Johansson B. Percutaneous pyelolithotomy. A new extraction technique. Scand J Urol Nephrol 1976;10:257-9.
6. De la Rossette JJ, Opondo D, Daels FP, Giusti G, et al. Categorisation of complications and validation of the Clavien score for percutaneous nephrolithotomy. Eur Urol. 62 (2012); 246-255

7. Myatt A, Palit $\mathrm{V}$, et al, The Uro-Clavien-Dindo system - will the limitations of the Clavien-Dindo System for grading complications of urological surgery allow modification of the classification to encourage national adoption within the UK? Br J Med Surg Urol. 2012;5:54-60.

8. Labate G, Modi P, et al, The Percutaneous Nephrolithotomy Global Study: Classification of complications. J Endorol 2011;8:1275-1280.

9. Fuller A, Razvi H, et a.; The CROES Percutaneous Nephrolithotomy Global Study: The influence of body mass index on outcome; J Urol, 2012; Vol 198,138-144

10. Ceballos-López JE, Carvaja GR, et al. Factores asociados a complicaciones en pacientes sometidos a nefrolitotomía percutánea. Rev Mex Urol 2016;76(3);148-152.

\section{AVISO PARA LOS AUTORES}

Revista Mexicana de Urología tiene una nueva plataforma de gestión para envío de artículos: https://www.revisionporpares.com/index.php/RMUrol ahí podrá inscribirse a la base de datos administrada por el sistema Open Journal System (OJS) que ofrece las siguientes ventajas para los autores:

- Subir sus artículos directamente al sistema.

- Conocer, en cualquier momento, el estado de los artículos enviados, es decir, si ya fueron asignados a un revisor, aceptados con o sin cambios, o rechazados.

- Participar en el proceso editorial corrigiendo y modificando sus artículos hasta su aceptación final. 